\title{
Human apolipoprotein L1 interferes with mitochondrial function in Saccharomyces cerevisiae
}

\author{
MOUNIA CHIDIAC ${ }^{12^{*}}$, JALIL DAHER $^{3^{*}}$, MÉLANIE BOECKSTAENS $^{4}$, PHILIPPE POELVOORDE $^{1}$, \\ BASSAM BADRAN $^{2}$, ANNA MARIA MARINI $^{4}$, ROY KHALAF $^{5}$ and LUC VANHAMME ${ }^{1}$
}

${ }^{1}$ Laboratory of Molecular Parasitology, Laboratory of Gene Molecular Biology, IBMM, Université Libre de Bruxelles, 6041 Gosselies, Belgium; ${ }^{2}$ Department of Biochemistry, Laboratory of Immunology, Lebanese University,

Faculty of Sciences, P.O. Box 6573, Hadath-Beirut; ${ }^{3}$ Department of Biology, University of Balamand, P.O. Box 100,

Tripoli, Lebanon; ${ }^{4}$ Laboratory of Membrane Transport Biology, IBMM, Faculty of Sciences, Université Libre de Bruxelles, 6041 Gosselies, Belgium; ${ }^{5}$ Department of Natural Sciences, Lebanese American University, P.O. Box 36, Byblos, Lebanon

Received October 20,2019; Accepted April 24, 2020

DOI: $10.3892 / \mathrm{mmr} .2020 .11271$

\begin{abstract}
To the best of our knowledge, the vertebrate apolipoprotein L (APOL) family has not previously been ascribed to any definite pathophysiological function, although the conserved $\mathrm{BH} 3$ protein domain suggests a role in programmed cell death or an interference with mitochondrial processes. In the present study, the human APOL1 was expressed in the yeast Saccharomyces cerevisiae in order to determine the molecular action of APOL1. APOL1 inhibited cell proliferation in a non-fermentable carbon source, such as glycerol, while it had no effect on proliferation in fermentable carbon sources, such as galactose. APOL1, expressed in yeast, is localized in the mitochondrial fraction, as determined via western blotting. APOL1 induced a loss of mitochondrial function, demonstrated by a loss of respiratory index, and mitochondrial membrane potential. Green fluorescent protein tagging of mitochondrial protein revealed that APOL1 was associated with abnormal mitochondrial and lysosomal morphologies, observed by a loss of the normal mitochondrial tubular network. Thus, the results of the present study suggest that APOL1 could be a physiological regulator of mitochondrial function.
\end{abstract}

Correspondence to: Professor Roy Khalaf, Department of Natural Sciences, Lebanese American University, Room 821, Block A, P.O. Box 36, Byblos, Lebanon

E-mail: roy.khalaf@lau.edu.lb

Professor Jalil Daher, Department of Biology, University of Balamand, Murr 108, UOB Street, P.O. Box 100, Tripoli, Lebanon E-mail: jalil.daher@balamand.edu.lb

${ }^{*}$ Contributed equally

Key words: apolipoprotein L1, yeast, mitochondrion, respiratory index, fermentation

\section{Introduction}

Apolipoprotein L (APOL) belongs to the high-density lipoprotein (HDL) family and is present in a number of species, including all mammals (1); however, its role remains poorly understood. The human APOL family consists of six genes clustered on chromosome 22 (2). Human APOL1 was first identified and characterized as one of the proteins associated with a subset of HDL particles (3). Its lipid binding capability was later demonstrated in vitro and is therefore considered to be involved in lipid transport and metabolism (4). Its toxicity against Trypanosoma brucei brucei was later demonstrated to involve a protein domain that can form anionic pores in lipid membranes (5). More recent studies have suggested novel actions for APOL1 in programmed cell death (PCD), more specifically autophagy. These actions have been shown to be associated with the BH3 domain, a Bcl2 homology domain; $\mathrm{BH} 3$ domain is ubiquitously expressed in regulators of programmed cell death in the $\mathrm{Bcl} 2$ and $\mathrm{BH} 3$-only families $(6,7)$.

The network of these regulators is complex, containing eight $\mathrm{BH} 3$-only and at least $20 \mathrm{Bcl} 2$ family members in humans, and is known to be involved in numerous types of cell death. As this machinery remains much simpler in yeast, involving only one Bcl2 family member, yBH3 (8), the present study used the yeast Saccharomyces cerevisiae as a model organism in order to investigate APOL1 function. In the present study, a yeast model for APOL1 expression was developed and used to investigate APOL1 function. The present study revealed its mitochondrial localization and interference with mitochondrial integrity, which had deleterious effects on yeast proliferation; the effect was observed when the cells were obliged to undergo respiration in a medium containing glycerol.

\section{Materials and methods}

Yeast strain and growth conditions. The $S$. cerevisiae strains used in the present study were isogenic to the wild type $\Sigma 1278 \mathrm{~b}$ strain: 23344c (ura3, lab collection: Laboratory of Membrane 
Transport Biology, IBMM, Université Libre de Bruxelles) and 27061b (ura3 and trp1, lab collection: Laboratory of Membrane Transport Biology, IBMM, Université Libre de Bruxelles) $(9,10)$. Cells were grown in a minimum buffered (pH 6.1) medium prepared as described previously (11) with $3 \%$ galactose (MGal), glucose (MGlu) or glycerol (MGly) as the carbon source or in non-inducible medium raffinose (MRaf) and glutamate as a nitrogen source, supplemented with vitamins and minerals (11). Yeasts were grown at $29^{\circ} \mathrm{C}$.

Plasmids and mutagenesis. For APOL1 expression, the human APOL1 was cloned into the centromeric p416Gal.1 (HA) (12) yeast expression plasmid under the control of a GAL1 promoter by homologous recombination. Yeast cells transformed with empty p416Gal.1 (HA) vector were used as control. The primers used are listed in Table I. The APOL with the mutated $\mathrm{BH} 3$ domain was amplified using PCR with Phusion $^{\mathrm{TM}}$ High-Fidelity DNA Polymerase (Thermo Fisher Scientific, Inc.) and the APOL1- $\triangle \mathrm{BH} 3 \mathrm{~F} / \mathrm{R}$ oligonucleotides (Table I) and introduced in the APOL1-pGal.1 vector. The reaction mixtures were processed with an initial denaturation period at $98^{\circ} \mathrm{C}$ for $30 \mathrm{sec}$, followed by a three-step PCR program for 30 cycles that consisted of $98^{\circ} \mathrm{C}$ for $10 \mathrm{sec}, 70^{\circ} \mathrm{C}$ for $20 \mathrm{sec}$ and $72^{\circ} \mathrm{C}$ for $25 \mathrm{sec}$, prior to a final extension step at $72^{\circ} \mathrm{C}$ for $10 \mathrm{~min}$.

The mutant APOL1- $\triangle \mathrm{BH} 3$ (BH3 domain deletion) was sequenced by Beckman Coulter (Illumina HiSeq) to verify the introduction of the desired substitution. pYX232-mtGFP (TRP1), encoding green fluorescent protein (GFP) fused to the mitochondrial pre-sequence subunit 9 of the $\mathrm{F}_{0}$-ATPase (mt-GFP) under the control of the constitutive triosephosphate isomerase promoter was used to monitor mitochondrial structure and morphology. pYX232-mtGFP was kindly provided by Professor Benedikt Westermann (Universität Bayreuth, Germany) (13).

Western blot analysis. Total protein extracts were performed as previously described (14). Aliquots of $1 \mathrm{ml}$ at an optical density (OD) of $0.2\left(10^{7}\right.$ cells $\left./ \mathrm{ml} ; \lambda=660 \mathrm{~nm}\right)$ from cells at the exponential growth phase were harvested by centrifugation at room temperature for $5 \mathrm{~min}$ at 3,500 x g. The cell pellet was suspended in $500 \mu \mathrm{l}$ water and the cells were lysed with $50 \mu \mathrm{l}$ of $2 \mathrm{M} \mathrm{NaOH}$ for $10 \mathrm{~min}$. The proteins were precipitated with $50 \mu \mathrm{l}$ of $50 \%$ trichloroacetic acid and collected by centrifugation at $4^{\circ} \mathrm{C}$ for $5 \mathrm{~min}$ at $12,000 \mathrm{x} \mathrm{g}$. The pellet was suspended with gel loading buffer containing $4 \%$ (w/v) SDS, $100 \mathrm{mM}$ Tris $\mathrm{pH} 6.8, \beta$-mercaptoethanol $2 \%(\mathrm{v} / \mathrm{v}), 20 \%(\mathrm{v} / \mathrm{v})$ glycerol and blue bromophenol. Samples were heat-treated at $37^{\circ} \mathrm{C}$ for $10 \mathrm{~min}$. For the western blotting analysis, equal protein amounts $(\sim 20 \mu \mathrm{g})$ were loaded onto a $8 \%$ SDS-PAGE. After transfer to nitrocellulose membranes, HA-tagged APOL1 was probed with anti-HA (1:10,000, cat. no. 26183, Molecular Probes; Thermo Fisher Scientific, Inc.), while Sc-Dpm1, Sc-Pma and Sc-porin were probed with anti-Dpm1 (1:5,000, cat. no. A-6429), anti-Pma1 (1:10,000, cat. no. MA1-91567) and anti-porin (1:5,000, cat. no. 459500; all from Invitrogen; Thermo Fisher Scientific, Inc.). Primary antibodies were detected with horseradish peroxidase-conjugated secondary antibodies anti-rabbit-IgG (1:1,000, cat. no. NA934; Cytiva) and anti-mouse-IgG (1:5,000, cat. no. NA931; Cytiva) for
$1 \mathrm{~h}$ at room temperature) followed by measurement of chemo-luminescence (Lumi-Light ${ }^{\text {PLus }}$, Roche Diagnostics). The western blot experiments in the present study analyzed membrane proteins; accordingly, Pmal was used as a suitable control (15).

Growth curve and clonogenic assay. For growth in liquid medium, yeast were grown overnight at $29^{\circ} \mathrm{C}$ in MGlu or MGal medium and diluted to an OD $(\lambda=660 \mathrm{~nm})$ of 0.2 in MGlu and MGal, respectively. OD was measured repeatedly over 9 days. In order to test the proliferation of cells grown on agar plates, yeast previously grown in liquid MGlu or MGal were spotted at a final concentration of $10^{3}$ cells on MGlu or MGal solid media. In parallel, aliquots were collected for immunostaining for the detection of APOL1/APOL1- $\triangle B H 3$ expression in inducible conditions.

Reactive oxygen species (ROS) assessment. Cells were cultured in MGal overnight at $29^{\circ} \mathrm{C}$ and then diluted in fresh media to an OD $(\lambda=660 \mathrm{~nm})$ of 0.2 . Cell aliquots were collected, washed twice in PBS and re-suspended at $1 \times 10^{7}$ cells $/ \mathrm{ml}$ in $1 \mathrm{ml}$ of $2.5 \mu \mathrm{g} / \mathrm{ml}$ dihydroethidium (DHE) (37291, Sigma-Aldrich) in PBS and incubated for $15 \mathrm{~min}$ in the dark at room temperature. Then, cells were washed with $1 \mathrm{ml}$ PBS and analyzed via flow cytometry. Flow cytometric analysis was performed on a Canto II (BD Biosciences) and results were analyzed with the FlowJo program (V10.5.0, FlowJo LLC).

Tetramethylrhodamine, ethyl ester (TMRE) staining. Yeast strain cell cultures were collected at $\mathrm{OD}=0.2(\lambda=660 \mathrm{~nm})$ of the exponential phase in MGal medium. Cells were incubated with 50 pM TMRE (TMRE-Mitochondrial Membrane Assay Kit, ab113852, Abcam) for $10 \mathrm{~min}$ at $4^{\circ} \mathrm{C}$, washed twice with PBS 1X, then cells were harvested, centrifuged $(1,600 \mathrm{x} \mathrm{g}$ for $4 \mathrm{~min}$ at $\left.4^{\circ} \mathrm{C}\right)$ and suspended $\left(\sim 1 \times 10^{6}\right.$ cells $\left.\mathrm{ml}^{-1}\right)$ in PBS, in order to be analyzed by flow cytometry, according to the manufacturer's protocol, on a Canto II (BD Biosciences). Carbonilcyanide $p$-triflouromethoxyphenylhydrazone (FCCP, ab120081, Abcam) treatment, an uncoupling agent, was used as a positive control, as it is able to completely depolarize the mitochondrial outer membrane. It was added to cell cultures at a final concentration of $20 \mu \mathrm{M} 20$ min prior to the incubation with TMRE.

Fluorescence microscopy for yeast organelles. For vacuole staining, cells were collected at $\mathrm{OD}=0.2(\lambda=660 \mathrm{~nm})$ of the exponential phase in MGAl growth medium and resuspended in $1 \mathrm{ml}$ of pre-heated medium and $80 \mathrm{nM}$ FM4-64 (T3166, Invitrogen, Thermo Fisher Scientific, Inc.). Cells were incubated at $29^{\circ} \mathrm{C}$ for $15 \mathrm{~min}$, centrifuged $(5,000 \mathrm{x} \mathrm{g}$ at room temperature for $5 \mathrm{~min}$ ), collected and resuspended in $5 \mathrm{ml}$ of medium and incubated at $29^{\circ} \mathrm{C}$ for $120 \mathrm{~min}$. For fixed $(1.1 \mathrm{ml}$ of $37 \%$ formaldehyde incubated at $29^{\circ} \mathrm{C}$ for $30 \mathrm{~min}$ ) or live cells, mt-GFP was monitored at a wavelength of $510 \mathrm{~nm}$ (magnification, x63 and x100).

Immunofluorescence assay. Yeast strains were cultured at $29^{\circ} \mathrm{C}$ overnight in MGal media until they reached the $\log$ growth phase. At $\mathrm{OD}=0.2(\lambda=660 \mathrm{~nm})$, cell aliquots of $9 \mathrm{ml}$ were fixed with $1.1 \mathrm{ml}$ of formaldehyde $37 \%$ and incubated at 
Table I. Primers used for construction of plasmids.

Oligonucleotides

or primers

Sequence, $5^{\prime}-3^{\prime}$

Purpose

\begin{tabular}{lll}
\hline APOL1-F & GTTAATATACCTCTATACTTTAACGTCAAGGAGAAAAAACTATAGGTAC & Plasmid construction \\
& CTAGATGGAGGGAGCTGCTTTGC3 & \\
APOL1-R & CAGCACCGGCTGCTCCTGCTCCTGCTCCTGCTCCTGCTCCCTCGAGCA & Plasmid construction \\
& GTTCTTGGTCCGCC & \\
APOL1- $\triangle \mathrm{BH} 3-\mathrm{F}$ & TTGTGGACCTTCCTTCTTATGTTATCCTCAAGC & Plasmid construction \\
APOL1- $\triangle \mathrm{BH} 3-\mathrm{R}$ & ATAAGAAGGCTCAAGGTCCACAAAGGCACCAC & Plasmid construction \\
\hline
\end{tabular}

$29^{\circ} \mathrm{C}$ for $30 \mathrm{~min}$. Cells were collected, washed and resuspended in Buffer B [Sorbitol (1 M), $\mathrm{K}_{2} \mathrm{HPO}_{4}, 3 \mathrm{H}_{2} \mathrm{O}(1 \mathrm{M})$ and $\mathrm{KH}_{2} \mathrm{PO}_{4}$ $(1 \mathrm{M})$ at $\mathrm{pH} 7.5]$. A total of $5 \mu \mathrm{l}$ of lyticase $(10,000 \mathrm{u} / \mathrm{ml})$ and $2 \mu \mathrm{l}$ of $\beta$-mercaptoethanol were added. After $30 \mathrm{~min}$ incubation at $29^{\circ} \mathrm{C}$, cells were collected, washed and suspended in $1 \mathrm{ml}$ of PBS 1X. A total of $20 \mu \mathrm{l}$ of yeast culture was seeded on 10-well slides (MP Biomedicals, LLC.). Cells were stained for APOL1/APOL1- $\triangle \mathrm{BH} 3$ expression levels with mouse anti-HA (1:100, cat. no. 26183, Molecular Probes; Thermo Fisher Scientific, Inc.) overnight at room temperature in a humidified chamber. A series of PBS washes preceded the secondary anti-mouse antibody (1:1,500, A-11001; Invitrogen; Thermo Fisher Scientific, Inc.) incubation for $1 \mathrm{~h}$ at room temperature in a humid chamber. DAPI (cat. no. D9542; Sigma Aldrich) was used to stain the nuclei of yeast cells. The revelation of APOL1/APOL1- $\triangle \mathrm{BH} 3$ expression was assessed using a Zeiss inverted fluorescence microscope (magnification, x63 and $\mathrm{x} 100$ ).

Mitochondrial isolation. The procedure was performed as previously described (16). Yeast strains were grown at $29^{\circ} \mathrm{C}$ for $16 \mathrm{~h}$, collected by centrifugation $(3,000 \mathrm{x} \mathrm{g}$ for $5 \mathrm{~min}$ at room temperature) and then washed twice with distilled water. Cells were suspended in dithiothreitol (DTT) buffer (100 mM Tris, pH 9.4, $10 \mathrm{mM}$ DTT) and incubated at $29^{\circ} \mathrm{C}$ for 20 min with gentle shaking. After removing the supernatant by centrifugation at $3,000 \mathrm{x} \mathrm{g}$ for $5 \mathrm{~min}$ at room temperature, cells were resuspended in Zymolyase buffer (1.2 M sorbitol buffer containing $1 \mathrm{mg}$ zymolyase $20 \mathrm{~T}$ per gram of cells). Following incubation at $29^{\circ} \mathrm{C}$ for $30 \mathrm{~min}$ with gentle shaking, cells were centrifuged at 2,200 $\mathrm{x}$ g for $8 \mathrm{~min}$ and resuspended in homogenization buffer [0.6 $\mathrm{M}$ sorbitol, $1 \mathrm{mM}$ EDTA, $10 \mathrm{mM}$ Tris, $0.2 \%$ (w/v) BSA pH 6.0]. These cells were centrifuged at 2,200 x g for $8 \mathrm{~min}$ at $4^{\circ} \mathrm{C}$ and then homogenized 15 times with a tight pestle in a glass homogenizer. The unbroken cells were then pelleted at $1,500 \mathrm{x} g$ for $5 \mathrm{~min}$ at $4^{\circ} \mathrm{C}$. The resulting supernatant was collected and centrifuged first at $3,000 \mathrm{xg}$ for $5 \mathrm{~min}$ at $4^{\circ} \mathrm{C}$ and again at $12,000 \mathrm{x} \mathrm{g}$ for $15 \mathrm{~min}$ at $4^{\circ} \mathrm{C}$. After repeating centrifugation $(3,000 \times \mathrm{g}$ for $5 \mathrm{~min}$ and $12,000 \mathrm{x} \mathrm{g}$ for $15 \mathrm{~min}$ at $4^{\circ} \mathrm{C}$ ) and resuspension of this pellet to yield pure mitochondria, the mitochondrial pellet was finally resuspended in SEM buffer (10 mM MOPS, $250 \mathrm{mM}$ sucrose, $1 \mathrm{mM}$ EDTA pH 7.4). Highly pure enriched mitochondria fraction was subjected to a sucrose gradient purification. After centrifugation of $134,000 \times \mathrm{g}$ for $1 \mathrm{~h}$ at $4^{\circ} \mathrm{C}$, an oxidized band was extracted and pelleted again at
$10,000 \mathrm{x} \mathrm{g}$ for $30 \mathrm{~min}$ at $4^{\circ} \mathrm{C}$. Western blot analyses were performed to examine preparation of the mitochondrial fraction as detailed above.

Index of respiratory competence assay (IRC). Exponentially MGal-growing cells at a final density of $10^{3}$ cells were spread onto MGly agar plates or MGal in parallel, incubated at $29^{\circ} \mathrm{C}$ for three days. At the end of the incubation, cells were observed and counted manually, and. the IRC was calculated as colony number observed on MGly plates divided by the number of colonies on MGal plates.

Statistical analysis. PRISM (version 6.0 GraphPad Prism software, Inc.) was used to perform statistical data analysis and plot the graphs. Data are presented as the mean \pm standard error of the mean (SEM). A minimum of three experiments were performed for statistical analysis. One-way ANOVA followed by Tukey's post hoc test and Student's t-test were used to determine statistically significant differences between the different experimental conditions. $\mathrm{P}<0.001$ was considered to indicate a statistically significant difference.

\section{Results}

APOL1 does not inhibit yeast proliferation in fermentable media. In order to investigate the action of human APOL1 in yeast, a sequence covering the whole APOL1 open reading frame was inserted in the yeast vector (pGAL1) allowing expression induction by galactose and repression by glucose. As the BH3 domain is one of the primary features of APOL1 and has been associated with the pro-apoptotic function of $\mathrm{Bcl} 2$ family proteins, the activity of the $\mathrm{BH} 3$-deleted version of APOL1 $(\triangle \mathrm{BH} 3)$ was tested. Cells were cultured in a medium containing glucose (MGlu-repressive conditions, negative control) or galactose (MGal-induced conditions) as carbon sources. In order to test cell proliferation, cells expressing APOL1 variants and control cells were plated on MGlu and MGal. No significant differences were observed in the number of colonies between cells expressing or not expressing APOL1 (Fig. 1B). As expected, no significant difference was observed between the different cells on MGlu, the repressive medium. Similarly, Fig. 1C shows no significant differences in the proliferation of cells expressing or not expressing an APOL1 variant on liquid MGal or MGlu media although its expression was properly induced in the MGal medium (Fig. 1A). Thus, APOL1 expression 

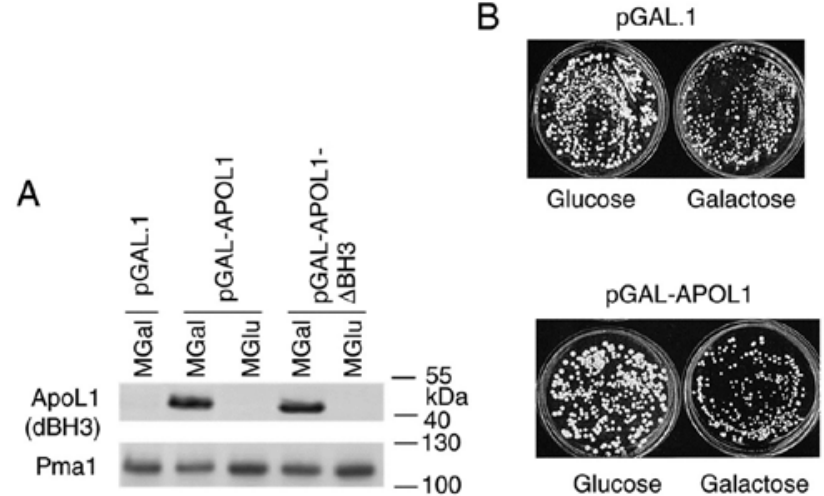

pGAL-APOL1- $\triangle \mathrm{BH} 3$

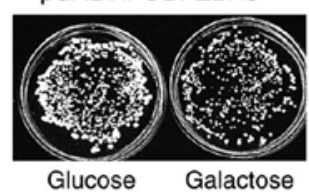

D

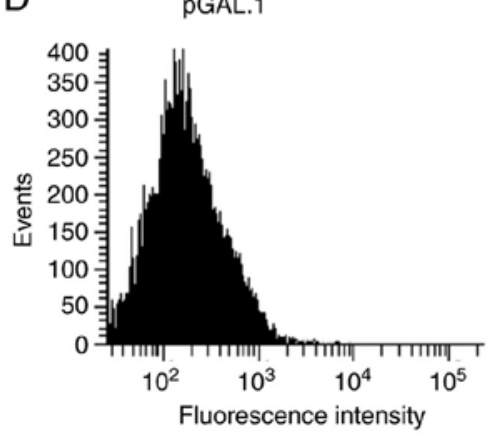

C
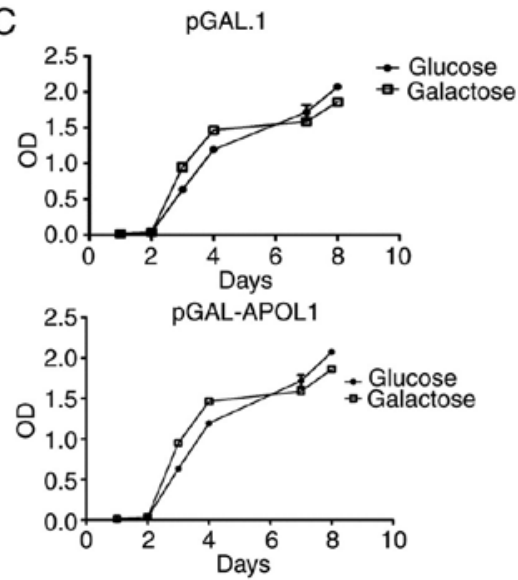

pGAL-APOL1- $\triangle \mathrm{BH} 3$

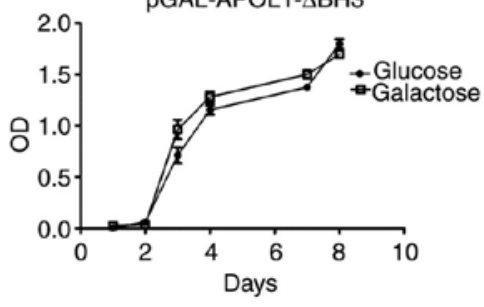

pGAL-APOL1- $\triangle \mathrm{BH} 3$

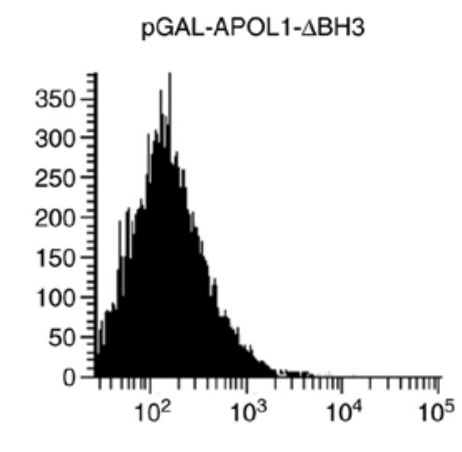

$\mathrm{E}$
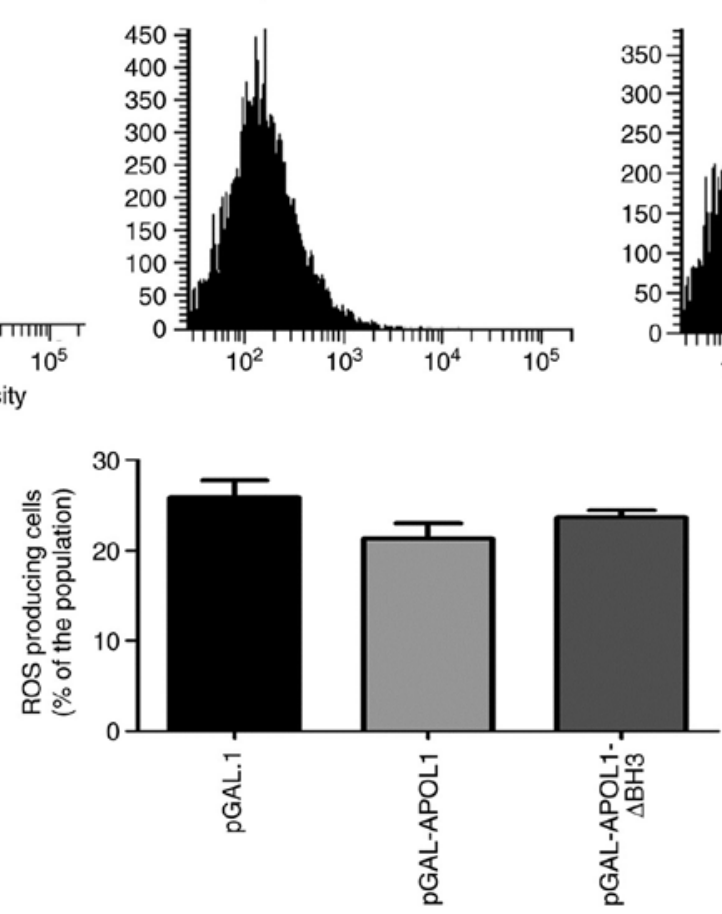

Figure 1. APOL1 expression levels, cell proliferation and intracellular ROS. (A) HA tagged wild type APOL1 and BH3-deleted APOL1 ( $\triangle \mathrm{BH} 3$ ) expression was induced in cells from a galactose inducible vector and revealed by immunoblotting assay using an anti-HA antibody; cells transfected with the empty vector (pGAL1) were used as negative control; loading control was made by immunoblotting analysis using an anti-Pma1 antibody. (B) APOL1 had no effect on yeast proliferation as measured using clonogenic assay: Cells analyzed in panel A were grown on solid media containing glucose or galactose and counted. (C) APOL1 had no effect on yeast proliferation as measured using liquid growth assay: Cells analyzed in panel A were inoculated in liquid media containing either glucose or galactose and proliferation was assessed by OD measurement. (D) Cells were treated with the ROS detecting reagent (dihydroethidium) and analyzed by flow cytometry. (E) Quantification of the flow cytometry analysis performed in D. Data are presented as the average of three independent cultures. Error bars represent the standard error of the mean. APOL1, apolipoprotein L 1; ROS, reactive oxygen species; OD, optical density.

levels do not influence cell proliferation in these conditions. In the presence of glucose or galactose, yeast cells primarily undergo anaerobic fermentation, generating ROS. In order to monitor ROS generation in induced and non-induced conditions, cells were stained with DHE detecting superoxide molecules and analyzed by flow cytometry in the present study. As presented in Fig. 1D and quantified in Fig. 1E, this analysis did not detect any significant difference in the fraction of the cell population producing ROS between the strains expressing or not APOL1. 
A
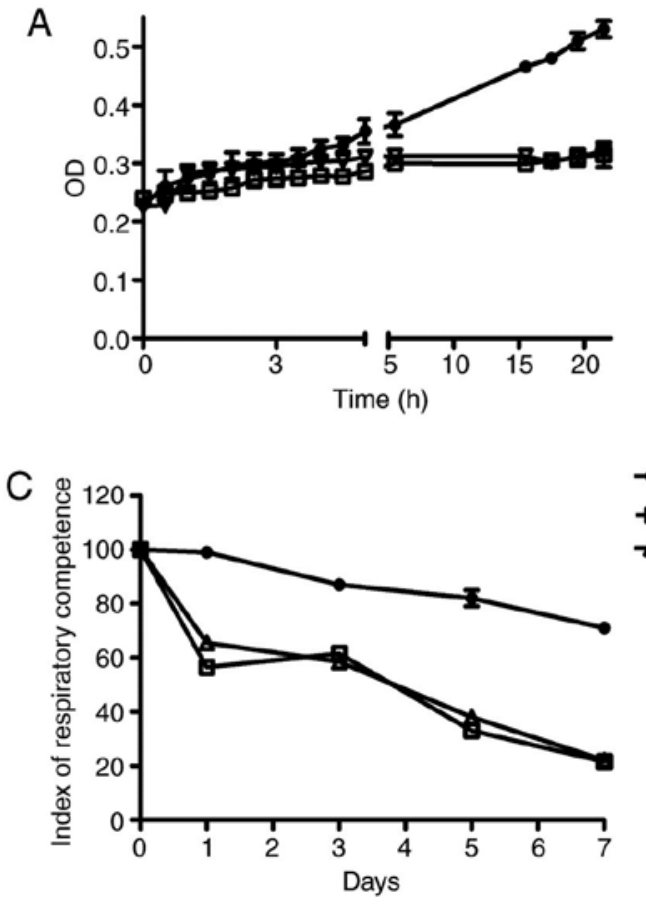

$\rightarrow$ pGAL1

\# PGAL-APOL1

$\leftarrow$ PGAL-APOL1- $\triangle \mathrm{BH} 3$
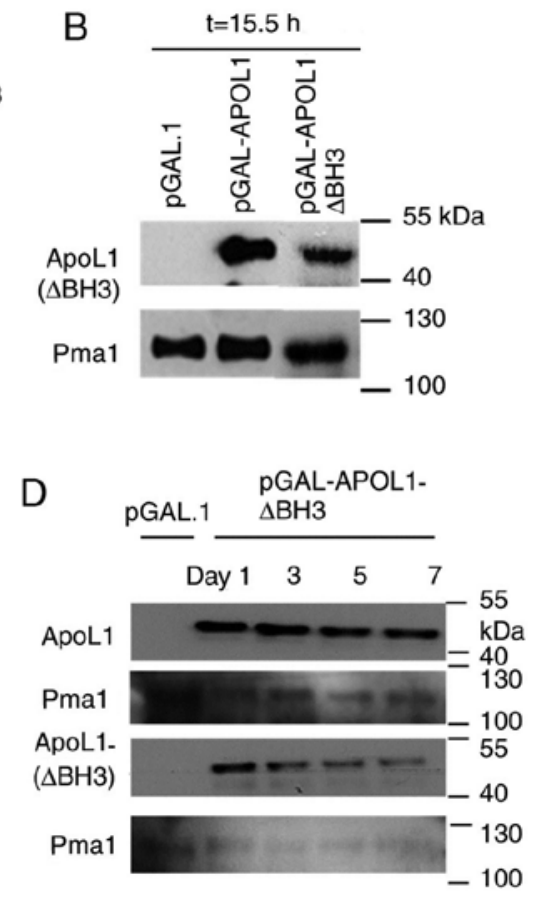

Figure 2. APOL1 expression levels and cell proliferation on non-fermentable media. (A) APOL1 inhibited yeast proliferation in non-fermentable media: HA tagged wild type and BH3-deleted $(\triangle \mathrm{BH} 3)$ APOL1 expression was induced by galactose and cells were then shifted in liquid glycerol containing MGly media at $\mathrm{T}=0$. Error bars represent standard errors on three independent experiments. (B) APOL1 protein expression levels after 15.5 h: Cell lysates were analyzed using immunoblotting with an anti-HA antibody. Loading control was made using immunoblotting analysis with an anti-Pma1 antibody. (C) APOL1 interfered with the respiratory competence. The capability of carbon sources to support proliferation was analyzed by plating cells (at 103 density) on solid media containing galactose or glycerol. Viable colonies on MGly plates were counted and compared with viable colonies under fermentable conditions. (D) APOL1 and $\mathrm{BH} 3$ deleted-APOL1 $(\triangle \mathrm{BH} 3)$ proteins were expressed during the index of respiratory competence assay (days $1,3,5$ and 7$)$. Cell extracts taken at the indicated time points were analyzed using immunoblotting with the indicated antibodies. Loading control was made using immunoblotting analysis with an anti-Pma1 antibody. APOL1, apolipoprotein L 1; MGly, glycerol-containing medium; OD, optical density; T, time.

APOL1 inhibits yeast proliferation in non-fermentable media. In the presence of a fermentable carbon source such as glucose or galactose, yeast cells preferentially generate energy by undergoing fermentation, and can grow normally with a minimal level of mitochondrial respiration, generating ethanol as the end product of fermentation. When fermentable carbon sources become limiting, genes required for respiration are induced, and ATP is generated by metabolizing non-fermentable carbon sources such as glycerol, ethanol or lactate in the mitochondria (17).

In order to determine whether human APOL1 could interfere with mitochondrial function, cells initially grown on MGal to induce APOL1 expression were transferred to an MGly medium, containing glycerol, a non-fermentable carbon source. As presented in Fig. 2A, yeast proliferation was inhibited in MGly media when APOL1 was induced (Fig. 2B) compared with the control culture. This indicated that APOL1 was able to inhibit yeast proliferation in conditions in which aerobic respiration is required, indicating that this protein could interfere with mitochondrial function.

As mitochondria are primarily involved in respiration, the present study assessed the IRC in cells expressing or not APOL1 variants. This index measures the percentage of viable respiration-competent cells and reflects the fraction of cells that can grow on non-fermentable (glycerol) carbon sources compared with the fraction of cells growing on fermentable (galactose) carbon sources (18). At the same time, the expression levels of the induced gene were verified (Fig. 2D). As presented in Fig. 2C, while the IRC value was $100 \%$ at the beginning of the experiment for all three strains, the IRC value started decreasing at day 1 only in the cells expressing APOL1 and APOL1 $\triangle \mathrm{BH} 3$. This decrease continued, reaching almost $20 \%$ after 7 days, while IRC remained higher than $70 \%$ for the control strain. The absence of the BH3 domain in APOL1 $\triangle \mathrm{BH} 3$ expressing cells had no effect on the number of viable respiration-competent cells.

APOL1 depolarizes the mitochondrial membrane. As a result of the cell proliferation inhibition observed on MGly media and the drop in respiratory index, the present study investigated whether the mitochondrial membrane potential $(\Delta \psi \mathrm{m})$ of yeast is affected by APOL1 induction. Maintenance of the membrane potential is key for mitochondrial functions (19). Thus, cells expressing or not expressing native APOL1 or APOL1- $\triangle \mathrm{BH} 3$ on a fermentable carbon source (MGal), were incubated with TMRE, a chemical dye able to accumulate in the mitochondria depending on membrane polarization (20). The positive control, which included treatment with FCCP (an ionophore that uncouples the electron transport chain from ATP production), completely prevented TMRE staining. As presented in Fig. 3, cells expressing APOL1, whether native or $\triangle B H 3$, showed a loss of membrane potential. TMRE uptake into the mitochondria was decreased by 68 and $70 \%$ in cells expressing native APOL1and $\triangle B H 3$ APOL1 respectively $(\mathrm{P}<0.0001)$. This suggests that APOL1 expression levels promote loss of mitochondrial membrane 

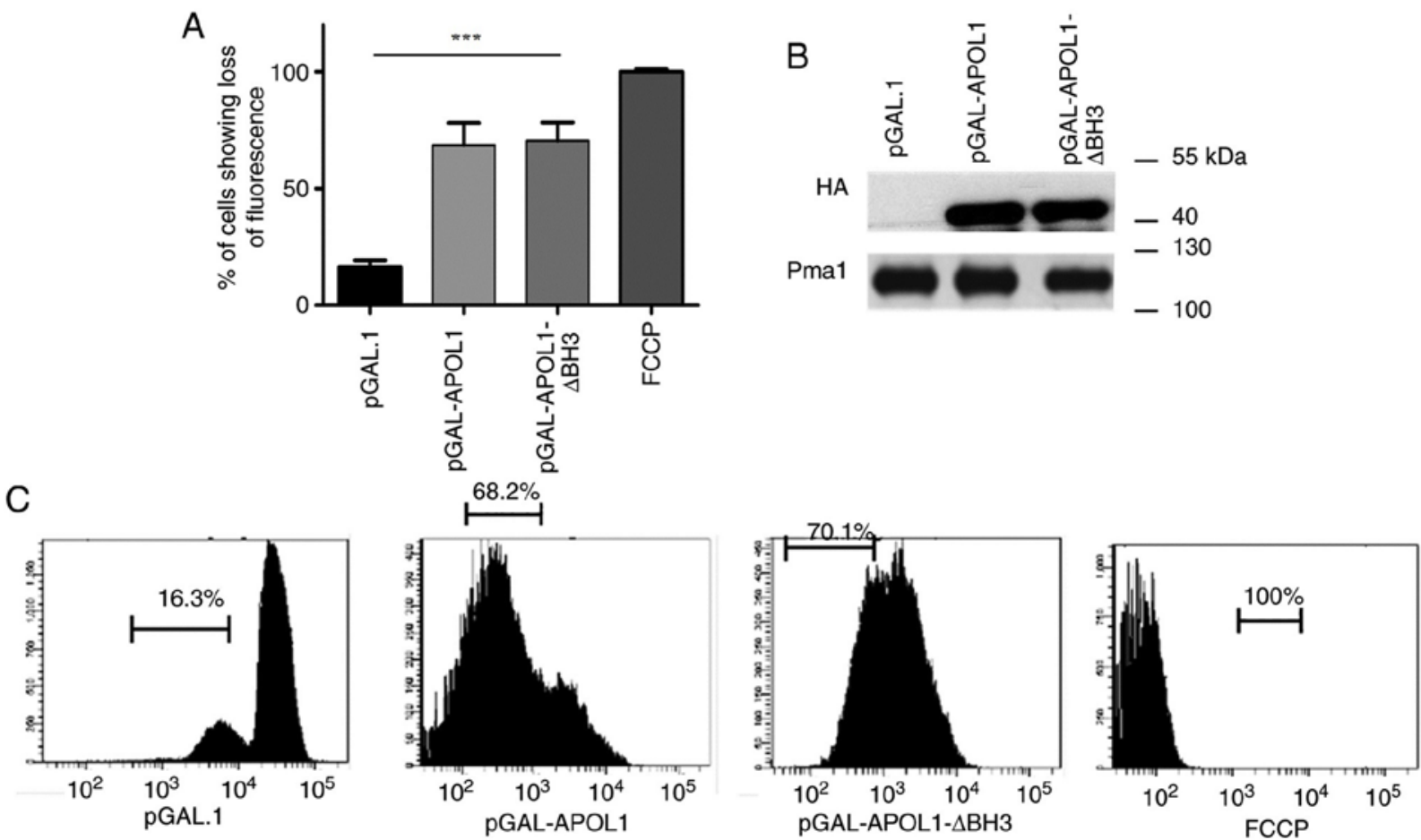

Figure 3. APOL1 expression levels and mitochondrial membrane depolarization. (A) APOL1 depolarized the mitochondrial membrane. Cells were incubated with TMRE; total fluorescent and non-fluorescent cells were analyzed using flow cytometry assays and the percentage of non-fluorescent cells in the population was plotted. Experiments were performed in liquid MGal media. Error bars represent standard errors of the mean in three independent experiments. (B) Simultaneously with flow cytometry assays, cell lysates were prepared and analyzed using immunoblotting with an anti-HA antibody. Loading control was made using immunoblotting analysis with an anti-Pmal antibody. (C) Cells were incubated with TMRE and analyzed using flow cytometry. FCCP was used as a positive control. $\mathrm{P}<0.001\left({ }^{* * *}\right)$ was considered statistically significant. APOL1, apolipoprotein L 1; TMRE, tetramethylrhodamine, ethyl ester; MGal, galactose-containing medium; FCCP, carbonilcyanide p-triflouromethoxyphenylhydrazone.

potential and that this ability does not require the $\mathrm{BH} 3$ domain.

APOL1 is found in the mitochondrial fraction. As APOL1 induction provoked mitochondrial membrane depolarization, the present study investigated a potential direct APOL1 interaction within the yeast mitochondria. In order to assess the subcellular localization of APOL1 in yeast, APOL1-expressing cells were grown on galactose medium for $24 \mathrm{~h}$ and mitochondria enrichment was performed using these cells. A fraction of HA-APOL1 was consistently found in the mitochondrial-enriched fraction (enriched mitochondria) as validated by the detection of the outer membrane mitochondrial channel-forming protein porin in this fraction (Fig. 4).

APOL1 induces mitochondrial morphological alterations in yeast cells. As APOL1 localizes in the mitochondria and interferes with proper function, the present study investigated whether their morphology was also affected. Morphology was visualized using mt-GFP, a mitochondria-targeted GFP protein. Cells co-expressing native APOL1 or APOL1- $\triangle \mathrm{BH} 3$ with mt-GFP were grown in MGal. In control cells not expressing APOL1, a normal branched tubular mitochondrial network located below the cell cortex (21) was observed. In contrast, in yeast cells expressing APOL1, the mitochondrial network was altered; the branched network disappeared and fluorescent patches appeared predominantly at the periphery of some of the cells; the mitochondrial signal in APOL1-expressing cells

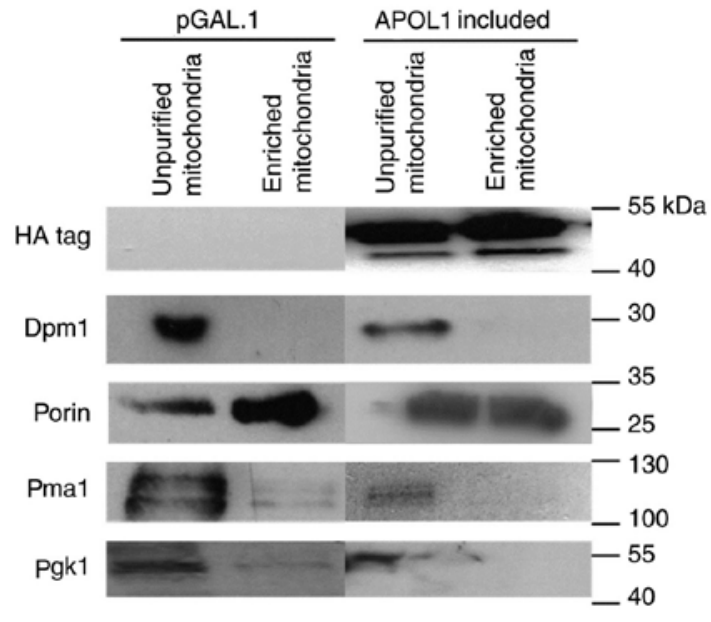

Figure 4. APOL1 subcellular localization. Extracts taken from cells transformed with the pGAL-1 vector expressing wild type APOL1 or the empty vector were fractionated. The UM and EM fractions are shown. These fractions were analyzed using western blotting with the following antibodies: Anti-HA, anti-Dpm1, anti-porin, anti-Pma1 and anti-PGK1. The UM fraction contains the endoplasmic reticulum, plasma membrane and mitochondrial fragments. The EM fraction is enriched for intact mitochondria. The figure shows non-adjacent bands from the same blot that were cut, adjusted and joined in order to avoid including non-relevant wells in the results. APOL1, apolipoprotein L 1; UM, unpurified mitochondria; EM, enriched mitochondria.

concentrated in fewer mitochondria that were larger compared with those in control cells and mt-GFP was not detectable in certain APOL1-expressing cells (Fig. 5A). 
A

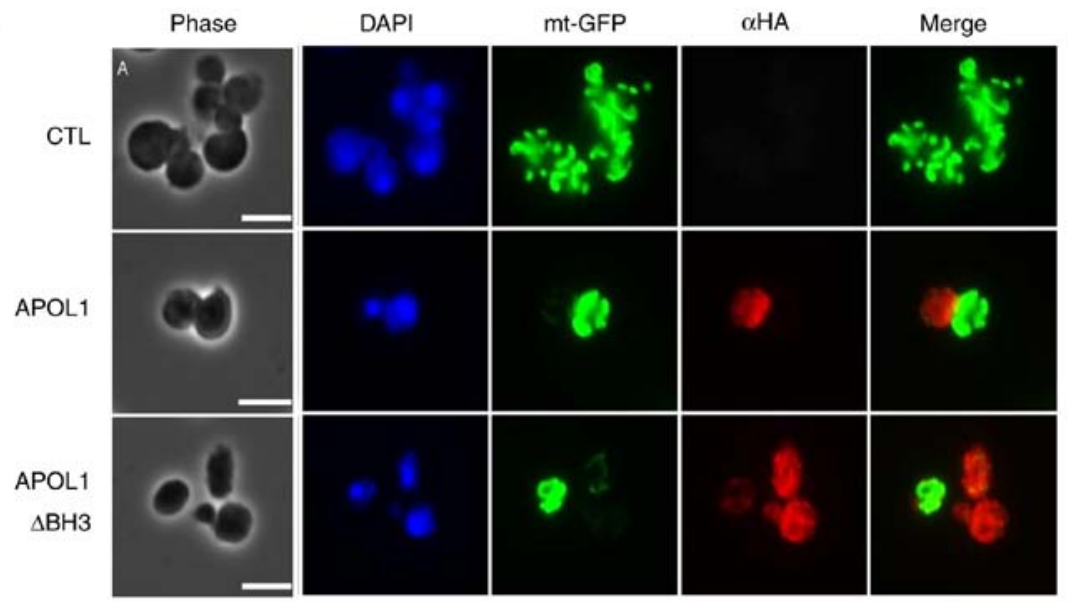

B

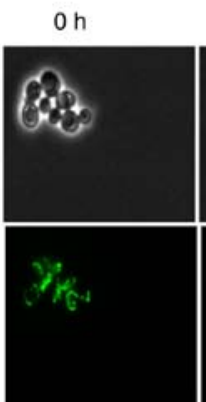

$\mathrm{Oh}$

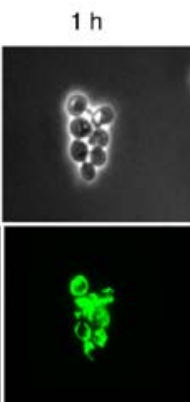

$1 \mathrm{~h}$

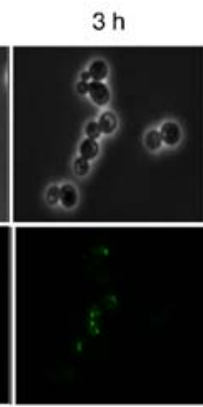

$3 \mathrm{~h}$

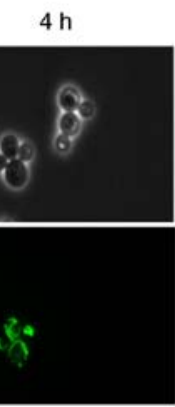

$4 \mathrm{~h}$

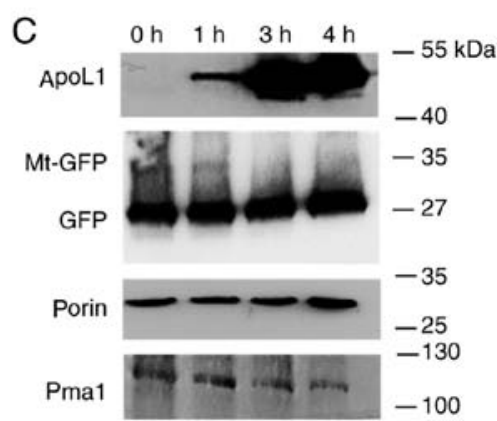

Figure 5. APOL1 expression levels and mitochondrial alterations. (A) APOL1 expression levels modified mitochondrial morphology as visualized by a mitochondrial marker. Cells were transformed by the plasmid pYX232-mtGFP, which expresses GFP fused to a mitochondrial matrix targeting sequence (mtGFP) alone (CTL) or together with plasmids expressing wild type APOL1 or BH3-deleted APOL1. Aliquots from cultures in MGal growth medium were then taken at OD 0.2 and treated with an anti-HA antibody (red), and stained with DAPI (blue). Magnification, x100. (B) Cells transformed by pYX232-mtGFP (with a constitutive promoter) and APOL1 expression vector were induced by galactose added to MRaf growth medium for the indicated times [time-dependent kinetics (0, 1, 3 and 4 h)]. (C) Immunoblot analysis of cell extracts from (B) demonstrated the expression levels of APOL1 (anti-HA), porin (Sc anti-porin) and GFP (anti-GFP). Scale bar, $5 \mu \mathrm{m}$. Magnification, x63. APOL1, apolipoprotein L 1; GFP, green fluorescent protein; MGal, galactose-containing medium; OD, optical density; MRaf, raffinose-containing medium.

In order to test if this morphological change was accompanied by a change in mitochondrial content, cells were grown on non-inducible and non-repressive medium (raffinose) and APOL1 was induced in a time-dependent manner by the addition of galactose to the growth medium. Using western blotting, the present study observed the stability of two mitochondrial components after inducing APOL1 for $4 \mathrm{~h}$ in the cells. Fig. 5C shows that HA-APOL1 (used as a control) was immunodetectable during the $4 \mathrm{~h}$ of induction. Porin, a mitochondrial outer membrane protein, remained stable during the $4 \mathrm{~h}$ induction. A decrease in the mitochondrial mt-GFP signal (35 KDa) was observed.

In order to further analyze these different structural patterns, the present study used a fluorescence microscopy assay during APOL1 induction kinetics. Cells were grown in MRaf and galactose was added at time $(\mathrm{T})=0$ to induce HA-APOL1 expression. At different times after the induction, cell aliquots were collected for mitochondrial visualization using mt-GFP as a marker and for immunoblot analysis. The present study identified different categories of the mitochondrial morphological structures that are consistent with what has been previously described (22). At $\mathrm{T}=0,92 \%$ of the cells contained the typical tubular shaped mitochondria (Fig. 6 and Table II). Mitochondria remained tubular in $90 \%$ of the cells at $(\mathrm{T})=0$. Following $1 \mathrm{~h}$ of APOL1 induction, $67.2 \%$ still contained a normal mitochondrial structure while 5\% had fragmented but widespread mitochondria, $12.9 \%$ showed mitochondria aggregated to one side of the cell, and $14.6 \%$ of cells lost the mitochondrial signal. In the control cells, mitochondria remained tubular throughout the experiment, while only $26.0 \%$ of APOL1-expressing cells displayed normal tubular mitochondria following $4 \mathrm{~h}$ of induction. At $\mathrm{T}=4$, a notable proportion of the cell population lacked detectable mitochondrial structure (42.5\%). This suggested that prolonged APOL1 expression levels may progressively modify mitochondrial structure.

As APOL1 uptake by African trypanosomes promotes lysosome swelling (23), the present study also observed the behavior of lysosome-like organelles, namely the vacuole, following APOL1 induction in yeast cells. Yeast cells were grown on MGal medium and stained with the fluorescent dye FM4-64, which accumulated at the vacuolar membrane. As presented in Fig. 7, APOL1-expressing cells exhibited alterations in vacuolar morphology, showing a vacuole fragmented into smaller vesicles compared with the control cells, which possessed a single, regular and large vacuole. These smaller vesicles restained as intensely as the single wild type vacuole. 
Table II. Cells were counted on the basis of different mitochondrial morphology: Mean count (\%) of yeast cells presenting the mitochondrial morphologies illustrated in Figure 6 at the indicated times. At least 100 cells were examined at each time point.

\begin{tabular}{cccccc}
\hline +APOL1 & Time & Normal $\%$ & Fragmented $\%$ & Aggregated $\%$ & No structure $\%$ \\
\hline $\mathrm{T}=0 \mathrm{~h}$ & 92 & - & - & 8 \\
$\mathrm{~T}=1 \mathrm{~h}$ & 67.2 & 5.1 & 12.9 & 14.6 \\
$\mathrm{~T}=3 \mathrm{~h}$ & 42.9 & 6 & 10.1 & 40.9 \\
$\mathrm{~T}=4 \mathrm{~h}$ & 26 & 12.2 & 19.1 & 42.5 \\
\hline
\end{tabular}
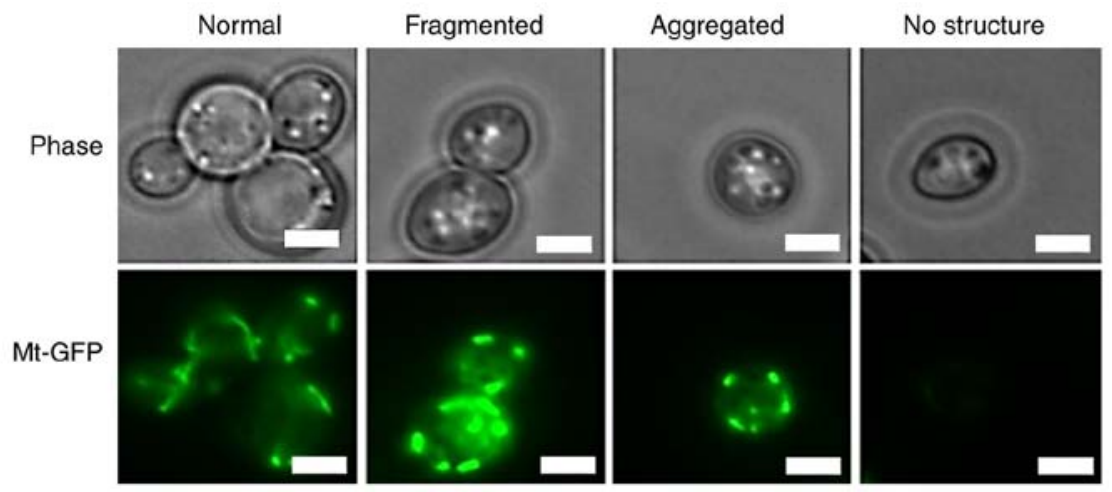

Figure 6. APOL1 expression levels and mitochondrial morphology. (A) APOL1 expression levels were induced using galactose for the indicated times [time-dependent kinetics $(0,1,3$ and 4 h)] in exponentially growing yeast cells expressing mt-GFP. This resulted in different mitochondrial morphologies: Tubular, fragmented and aggregated mitochondria. APOL1, apolipoprotein L 1; mt-GFP, mitochondria-targeted green fluorescent protein. Scale bar, $5 \mu$ m. Magnification, x100.

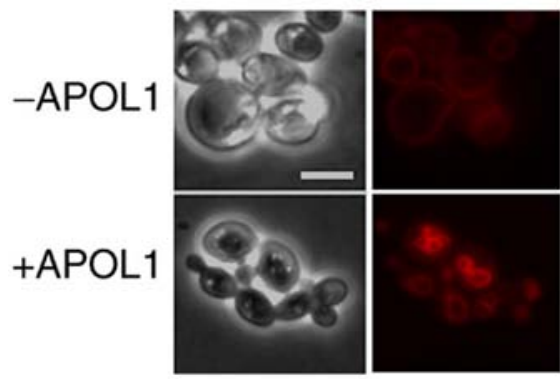

Figure 7. APOL1 expression levels and vacuole morphology. APOL1 expression levels were associated with vacuole fragmentation. APOL1 expression was induced (using galactose) in exponentially growing yeast cells for $12 \mathrm{~h}$. Cells were then stained with the vacuole-targeted dye FM 4-64. Representative images of control yeast cells (single large vacuoles) and cells expressing APOL1 (two to four medium-sized vacuoles) are shown. Scale bar, $5 \mu \mathrm{m}$. Magnification, x63. APOL1, apolipoprotein L 1.

\section{Discussion}

Previous studies have suggested an association between certain APOL family members and PCD of neutrophils in chronically ill patients and murine dendritic cells $(24,25)$. As PCD is much simpler in yeast than in mammals, the present study used yeast to investigate the molecular action of APOL1. The APOL family is conserved in evolution as members are found in fish, but they are also divergent between mammals (4). For instance, APOL1 is specific to humans (26). The present study demonstrated that ectopic expression of human APOL1 in S. cerevisiae is associated with disruption of mitochondrial function, dissipation of the mitochondrial membrane potential and alteration of mitochondrial and vacuolar morphology. The present study further showed that APOL1 alters yeast respiratory function. APOL1 induction did not have any effect on cell proliferation in the presence of fermentable carbon source although it affected yeast proliferation in non-fermentable media, which was associated with mitochondrial membrane depolarization. As this was also associated with a drop in respiratory index, this proliferation defect may be ascribed to a loss of mitochondrial functionality that is only detrimental if fermentation is inhibited. Likewise, mutations that affect the mitochondria are known to affect yeast respiration capacity in MGly media (27). The association between loss of membrane potential and APOL1 induction may be the result of direct APOL1 action on the mitochondrial membrane, similar to the mitochondrial pore forming capability of the Bax and Bak members of the $\mathrm{Bcl} 2$ family. This pore-forming capacity is associated with multimerization mediated partly by the $\mathrm{BH} 3$ domain, a peptide sequence conserved in APOL1 (28). The observation that an APOL1 deleted on the $\mathrm{BH} 3$ domain was still able to interfere with mitochondrial function is in disagreement with this finding. On the contrary, the present study demonstrated that, in yeast, APOL1 localized at the mitochondria. This observation is in line with recent findings regarding the localization of APOL1 to structures surrounding the mitochondria in human podocyte cell lines where it also triggers a decrease in mitochondrial respiration (29). This suggests that the molecular associations required for this specific mitochondrial localization are conserved between mammalian and 


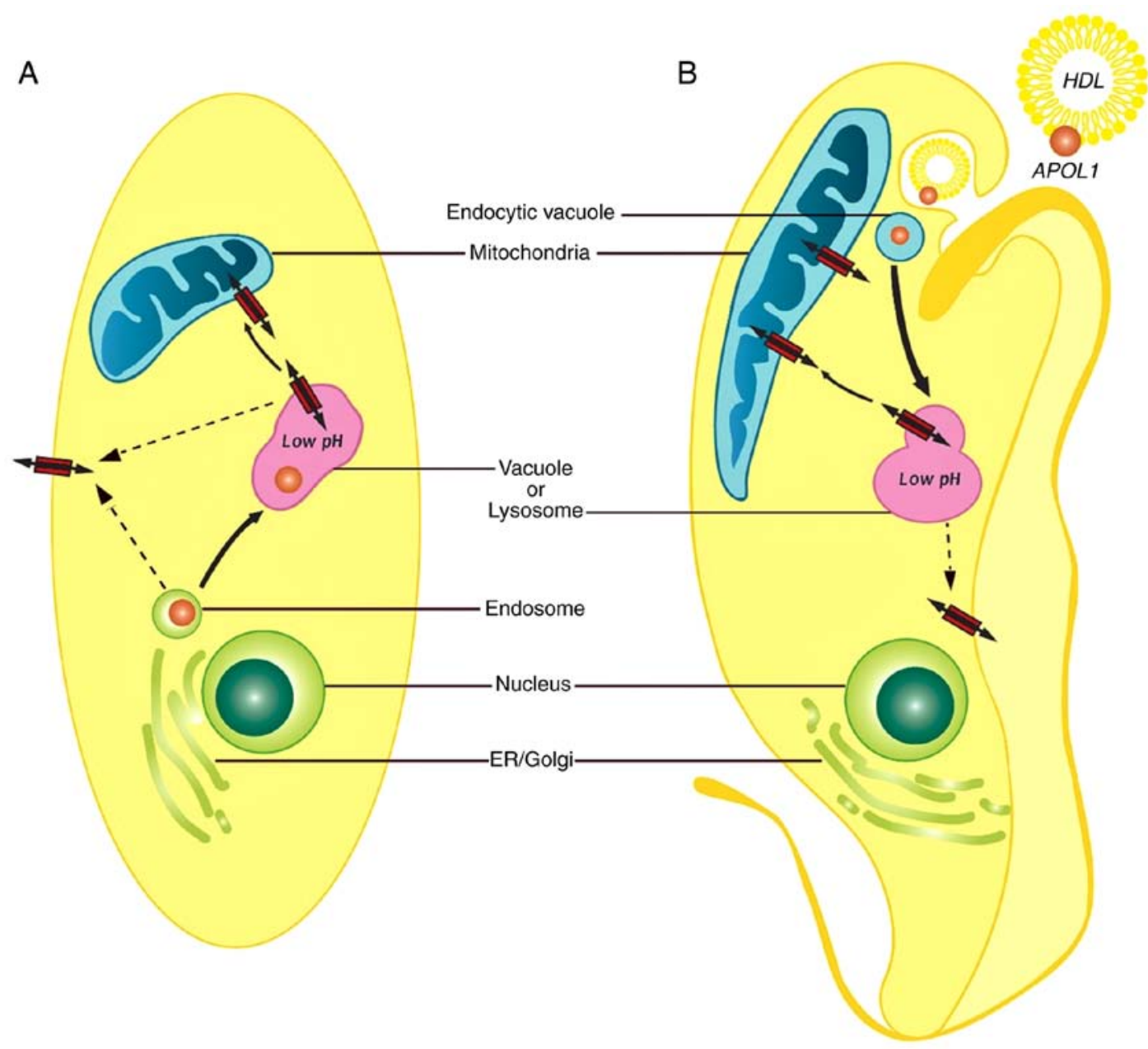

Figure 8. Model of APOL1-induced death. A model of intracellular APOL1 induced death that is associated with mitochondrial membrane permeabilization as well as changes in lysosomal and vacuolar acidification. (A) In yeast (and human kidney cells), APOL1 (orange) may cause changes in mitochondrial membrane potential and vacuolar acidification, ultimately resulting in cell hypertrophy and death. (B) In trypanosomes, during the course of trypanolysis, APOL1 localizes to the lysosomal membrane causing lysosomal membrane permeabilization and to the mitochondrial membrane causing depolarization of the mitochondria. The two processes result in cell lysis and death. APOL1, apolipoprotein L 1; HDL, high-density lipoprotein; ER, endoplasmic reticulum.

yeast mitochondria. APOL1 intracellular function may regulate the outer mitochondrial membrane potential in certain circumstances. This is suggested by the fact that APOL1 can induce lysosomal and mitochondrial membrane permeabilization in the distant Trypanosoma brucei eukaryote (22). Ongoing studies are attempting to elucidate the molecular requirements using a battery of mutants affecting different components of mitochondrial metabolism and cell death. The effects of APOL1 also seem to extend to the vacuole. It is not clear how APOL1 exerts this effect but it is known that Bax expressed in yeast cells causes vacuoles to fragment. Vacuole fragmentation is also induced by certain mutants, such as Vphlp (partial deletions of the V0 subunit of V-ATPase); Vph1p affects vacuole acidification (30). This APOL1 effect in yeast is similar to that previously found in trypanosomes; APOL1 lyses these protozoans, which involves localization in organelles, lysosome swelling and mitochondrial depolarization (31). This effect is also consistent with previous discoveries regarding the consequences of APOL1 high-risk variants (G1 and G2) on podocytes in people with chronic kidney diseases. It has been shown that APOL1 G1 and G2 variants were implicated in promoting cell damage by mediating endoplasmic reticulum stress, compromising lysosomal membrane permeability and disrupting trafficking processes including vacuole acidification $(32,33)$. The yeast model provides insights into the mode of action of mammalian APOL1; it suggests that APOL1 interacts with the mitochondrial and vacuolar membranes, causing alteration of mitochondrial and vacuolar morphology, disruption of their functions (primarily by depolarization of the mitochondrial membrane) and the disruption of trafficking processes that may involve changes in vacuolar $\mathrm{pH}$. The present study further showed that APOL1 alters yeast respiratory function, which is followed by a proliferation arrest when yeast cells are forced to use mitochondrial respiration as a unique source of energy. A combined genetics and biochemistry approach may identify the mechanisms that underlie the function of APOL1 and its role in mammalian cells. For example, APOL1 may be involved when mammalian cells shift from mitochondrial metabolism to glycolysis for energy production, a phenomenon encountered in numerous types of cancers (the Warburg effect), or during an immune reaction when $\mathrm{T}$ lymphocytes are actively proliferating. A model of how APOL1 induces cell death is outlined in Fig. 8. In yeast and human kidney 
cells, APOL1 is produced intracellularly by normal biosynthetic pathways. APOL1 may cause changes in mitochondrial membrane potential and vacuolar acidification, ultimately resulting in cell hypertrophy and death $(34,35)$. In trypanosomes, the process of trypanolysis starts with the uptake of HDL particles containing APOL1 by the parasite. As acidity increase within the endocytic compartments within the cell, APOL1 localizes to the lysosomal membrane causing lysosomal membrane permeabilization and osmotic swelling. In addition, acidified APOL1 can be transported to the mitochondrial membrane causing depolarization of the mitochondria. The two processes result in cell lysis and death (31). Although not examined in the present study, APOL1 may be inserted into plasma membranes of both trypanosomes and human kidney cells (36).

\section{Acknowledgements}

Not applicable.

\section{Funding}

The present study has been funded by research programs of the FNRS-FRS (Belgian National Fund for Scientific Research), Azm and Saadé Association (grant no. 110617) and AUF (Agence Universitaire de la Francophonie; grant no. 140909). Additional funding was provided by the Bureau of International Relations (grant no. bric98156) of the Université Libre de Bruxelles (ULB).

\section{Availability of data and materials}

All data generated or analyzed during this study are included in this published article.

\section{Authors' contributions}

$\mathrm{MC}, \mathrm{JD}, \mathrm{MB}$ and PP were involved in performing the experiments and analyzing the results. MC drafted the original manuscript. JD and LV wrote the manuscript. JD, MB, BB, AMM, LV and RK reviewed the manuscript. JD, LV and RK edited the manuscript. AMM and LV developed the methodology. LV and BB conceptualized the study. RK interpreted the data. All authors read and approved the final manuscript.

\section{Ethics approval and consent to participate}

Not applicable.

\section{Patient consent for publication}

Not applicable.

\section{Competing interests}

LV is Director of Research at the FNRS. AMM is Senior Research Associate at the FNRS and WELBIO investigator. $\mathrm{MB}$ is a scientific research worker supported by WELBIO.

\section{References}

1. Page NM, Butlin DJ, Lomthaisong K and Lowry PJ: The human apolipoprotein L gene cluster: Identification, classification, and sites of distribution. Genomics 74: 71-78, 2001.

2. Monajemi H, Fontijn RD, Pannekoek H and Horrevoets AJ: The apolipoprotein L gene cluster has emerged recently in evolution and is expressed in human vascular tissue. Genomics 79: 539-546, 2002.

3. Duchateau PN, Pullinger CR, Orellana RE, Kunitake ST, Naya-Vigne J, O'Connor PM, Malloy MJ and Kane JP: Apolipoprotein L, a new human high density lipoprotein apolipoprotein expressed by the pancreas. Identification, cloning, characterization, and plasma distribution of apolipoprotein $\mathrm{L}$. J Biol Chem 272: 25576-25582, 1997.

4. Vanhollebeke B and Pays E: The function of apolipoproteins L. Cell Mol Life Sci 63: 1937-1944, 2006.

5. Thomson R and Finkelstein A: Human trypanolytic factor APOL1 forms $\mathrm{pH}$-gated cation-selective channels in planar lipid bilayers: Relevance to trypanosome lysis. Proc Natl Acad Sci USA 112: 2894-2899, 2015.

6. Youle RJ and Strasser A: The BCL-2 protein family: Opposing activities that mediate cell death. Nat Rev Mol Cell Biol 9: 47-59, 2008.

7. Zhaorigetu S, Wan G, Kaini R, Jiang Z and Hu CA: ApoL1, a $\mathrm{BH} 3$-only lipid-binding protein, induces autophagic cell death. Autophagy 4: 1079-1082, 2008.

8. Büttner S, Ruli D, Vögtle FN, Galluzzi L, Moitzi B, Eisenberg T, Kepp O, Habernig L, Carmona-Gutierrez D, Rockenfeller P, et al: A yeast $\mathrm{BH} 3$-only protein mediates the mitochondrial pathway of apoptosis. EMBO J 30: 2779-2792, 2011.

9. Bechet J, Greenson $\mathrm{M}$ and Wiame JM: Mutations affecting the repressibility of arginine biosynthetic enzymes in Saccharomyces cerevisiae. Eur J Biochem 12: 31-39, 1970.

10. Springael JY, De Craene JO and André B: The yeast Npi1/Rsp5 ubiquitin ligase lacking its $\mathrm{N}$-terminal $\mathrm{C} 2$ domain is competent for ubiquitination but not for subsequent endocytosis of the Gap1 permease. Biochem Biophys Res Commun 257: 561-566, 1999.

11. Jacobs P, Jauniaux JC and Grenson M: A cis-dominant regulatory mutation linked to the $\operatorname{argB}-\operatorname{argC}$ gene cluster in Saccharomyces cerevisiae. J Mol Biol 139: 691-704, 1980.

12. Mumberg D, Müller R and Funk M: Regulatable promoters of Saccharomyces cerevisiae: Comparison of transcriptional activity and their use for heterologous expression. Nucleic Acids Res 22: 5767-5768, 1994.

13. Westermann B and Neupert W: Mitochondria-targeted green fluorescent proteins: Convenient tools for the study of organelle biogenesis in Saccharomyces cerevisiae. Yeast 16: 1421-1427, 2000.

14. Volland C, Urban-Grimal D, Géraud G and Haguenauer-Tsapis R: Endocytosis and degradation of the yeast uracil permease under adverse conditions. J Biol Chem 269: 9833-9841, 1994.

15. Boeckstaens M, Merhi A, Llinares E, Van Vooren P, Springael JY, Wintjens R and Marini AM: Identification of a novel regulatory mechanism of nutrient transport controlled by TORC1-Npr1-Amu1/Par32. PLoS Genet 11: e1005382, 2015.

16. Gregg C, Kyryakov P and Titorenko VI: Purification of mitochondria from yeast cells. J Vis Exp 30: e1417, 2009.

17. Piskur J, Rozpedowska E, Polakova S, Merico A and Compagno C: How did Saccharomyces evolve to become a good brewer? Trends Genet 22: 183-186, 2006.

18. Parrella E and Longo VD: The chronological life span of Saccharomyces cerevisiae to study mitochondrial dysfunction and disease. Methods 46: 256-262, 2008.

19. Crowley LC, Christensen ME and Waterhouse NJ: Measuring mitochondrial transmembrane potential by TMRE staining. Cold Spring Harb Protoc 2016, 2016.

20. Hoffmann HP and Avers CJ: Mitochondrion of yeast: Ultrastructural evidence for one giant, branched organelle per cell. Science 181: 749-751, 1973.

21. Pevala V, Kolarov $\mathbf{J}$ and Polčic P: Alterations in mitochondrial morphology of Schizosaccharomyces pombe induced by cell-death promoting agents. Folia Microbiol (Praha) 52: 381-390, 2007.

22. Vanwalleghem G, Fontaine F, Lecordier L, Tebabi P, Klewe K, Nolan DP, Yamaryo-Botté Y, Botté C, Kremer A, Burkard GS, et al: Coupling of lysosomal and mitochondrial membrane permeabilization in trypanolysis by APOL1. Nat Commun 6: 8078, 2015. 
23. Zamzami N, Marchetti P, Castedo M, Decaudin D, Macho A, Hirsch T, Susin SA, Petit PX, Mignotte B and Kroemer G: Sequential reduction of mitochondrial transmembrane potential and generation of reactive oxygen species in early programmed cell death. J Exp Med 182: 367-377, 1995.

24. Akl I, Lelubre C, Uzureau P, Piagnerelli M, Biston P, Rousseau A Badran B, Fayyad-Kazan H, Ezedine M, Vincent JL, et al: Apolipoprotein L expression correlates with neutrophil cell death in critically ill patients. Shock 47: 111-118, 2017.

25. Uzureau S, Coquerelle C, Vermeiren C, Uzureau $P$ Van Acker A, Pilotte L, Monteyne D, Acolty V, Vanhollebeke B, Van den Eynde B, et al: Apolipoproteins L control cell death triggered by TLR3/TRIF signaling in dendritic cells. Eur J Immunol 46: 1854-1866, 2016.

26. Francis BR, White KH and Thorsness PE: Mutations in the Atp1p and Atp3p subunits of yeast ATP synthase differentially affect respiration and fermentation in Saccharomyces cerevisiae. J Bioenerg Biomembr 39: 127-144, 2007.

27. Granado D, Müller D, Krausel V, Kruzel-Davila E, Schuberth C, Eschborn M, Wedlich-Söldner R, Skorecki K, Pavenstädt H, Michgehl U and Weide T: Intracellular APOL1 risk variants cause cytotoxicity accompanied by energy depletion. J Am Soc Nephrol 28: 3227-3238, 2017.

28. Baars TL, Petri S, Peters C and Mayer A: Role of the V-ATPase in regulation of the vacuolar fission-fusion equilibrium. Mol Biol Cell 18: 3873-3882, 2007.

29. Kruzel-Davila E, Shemer R, Ofir A, Bavli-Kertselli I, Darlyuk-Saadon I, Oren-Giladi P, Wasser WG, Magen D, Zaknoun E, Schuldiner M, et al: APOL1-mediated cell injury involves disruption of conserved trafficking processes. J Am Soc Nephrol 28: 1117-1130, 2017.

30. Lan X, Jhaveri A, Cheng K, Wen H, Saleem MA, Mathieson PW, Mikulak J, Aviram S, Malhotra A, Skorecki K and Singhal PC: APOL1 risk variants enhance podocyte necrosis through compromising lysosomal membrane permeability. Am J Physiol Renal Physiol 307: F326-F336, 2014.
31. Vanhamme L, Paturiaux-Hanocq F, Poelvoorde P, Nolan DP, Lins L, Van Den Abbeele J, Pays A, Tebabi P, Van Xong H, Jacquet A, et al: Apolipoprotein L-I is the trypanosome lytic factor of human serum. Nature 422: 83-87, 2003.

32. Wen H, Kumar V, Lan X, Shoshtari SSM, Eng JM, Zhou X, Wang F, Wang H, Skorecki K, Xing G, et al: APOL1 risk variants cause podocytes injury through enhancing endoplasmic reticulum stress. Biosci Rep 38: BSR20171713, 2018.

33. Greene AS and Hajduk SL: Trypanosome lytic factor-1 initiates oxidation stimulated osmotic lysis of Trypanosoma brucei brucei. J Biol Chem 291: 3063-3075, 2016.

34. Cheng D, Weckerle A, Yu Y, Ma L, Zhu X, Murea M, Freedman BI, Parks JS and Shelness GS: Biogenesis and cytotoxicity of APOL1 renal risk variant proteins in hepatocytes and hepatoma cells. J Lipid Res 56: 1583-1593, 2015.

35. Olabisi OA, Zhang JY, VerPlank L, Zahler N, DiBartolo S III, Heneghan JF, Schlöndorff JS, Suh JH, Yan P, Alper SL, et al: APOL1 kidney disease risk variants cause cytotoxicity by depleting cellular potassium and inducing stress-activated protein kinases. Proc Natl Acad Sci USA 113: 830-837, 2016.

36. Limou S, Dummer PD, Nelson GW, Kopp JB and Winkler CA: APOL1 toxin, innate immunity, and kidney injury. Kidney Int 88: 28-34, 2015.

This work is licensed under a Creative Commons Attribution-NonCommercial-NoDerivatives 4.0 International (CC BY-NC-ND 4.0) License. 